\title{
Harlequin Reaction
}

National Cancer Institute

\section{Source}

National Cancer Institute. Harlequin Reaction. NCI Thesaurus. Code C112829.

Transient blanching of the lower half of the body while in the lateral recumbent position, most commonly seen in premature or low birth weight infants. 